\title{
Influence of inclusion type and size on the fatigue strength of high strength steels
}

\author{
Jens Schumacher ${ }^{1,}$, Brigitte Clausen ${ }^{1}$, and Hans-Werner Zoch $^{1}$ \\ ${ }^{1}$ Leibniz Institute for Materials Engineering IWT, Badgasteiner Str. 3, 28359 Bremen, Germany
}

\begin{abstract}
In this investigation the influence of the inclusion type and size on the fatigue strength of different steels is analysed. As database case hardened, quenched and tempered as well as bearing steels in different heat treatment conditions, which have been investigated in several research projects over the last two decades, are used. Based on the approach of Murakami the local stress intensity at inclusions was determined to evaluate the influence of fracture causing inclusions on the fatigue strength. Different fatigue criteria have been used to calculate the local equivalent stress amplitudes considering residual stresses in the specimens, mean stresses during loading and multiaxial stresses in notched specimens. Since many run out specimens were subsequently fatigued at a higher stress amplitude, the critical inclusion type and size which have survived the initial stress amplitude could be determined. As a result the local stress intensity at inclusions which leads to no fatigue failure could be calculated as well. With the knowledge of the stress intensity factor range of the fractured and run out specimens a threshold stress intensity factor range could be derived for the different steels and inclusion sizes.
\end{abstract}

\section{Introduction}

In fatigue tests steel specimens fail at stresses below the static strength [1]. Cracks are formed either at the surface or in the interior of the specimen which propagate and lead finally to the fracture of the specimen. In case of internal failures inclusions frequently act as crack initiating sites, where oxides, nitrides and sulphides are the most frequently mentioned inclusion types [2-5]. It is well known that with increasing inclusion size the fatigue strength of steel specimens is reduced [6]. Different statements are published about the influence of the inclusion type on the fatigue strength. According to Murakami the inclusion type has nearly no influence on the fatigue strength. On the other hand, depending on the inclusion type, different harmful effects on fatigue strength are observed by other authors [7]. Hence, in this investigation the influence of the inclusion type and size on the fatigue strength of different steels is analysed. Different high strength steels in different heat treatment conditions, which have been investigated in several research projects over the last two decades, are used as database. A fracture mechanical approach was used to evaluate the influence of fracture causing inclusions on the fatigue strength. Different fatigue criteria have been used to calculate the local stress amplitude to consider residual stresses in the specimens, mean stresses during loading and multiaxial stresses in notched specimens. The threshold stress intensity factor range was determined separately for different inclusion types. For that reason the influence of the inclusion types oxide, sulphide, carbide and nitride on the fatigue strength of steels with varying hardness could be evaluated.

\section{Calculation approach}

For the calculation of the influence of the inclusion size and type on the fatigue strength of steel specimens a fracture mechanical approach is used. The inclusions are regarded as a crack with the same size as the inclusion in a plane perpendicular to the loading direction as described by Murakami [6].

According to Murakami the stress intensity factor range $\Delta K$ depends on the equivalent stress amplitude $\sigma_{\mathrm{ae}}$ and the size of the inclusion area $^{0.5}$ which equals the square root of the projected area of the inclusion in the fracture plane:

$$
\Delta K=\sigma_{\mathrm{ae}} \cdot \sqrt{\pi \sqrt{\text { area }}}
$$

The stress intensity factor range which leads to a fatigue failure of a specimen can be calculated with Equation 1 after the size of the crack initiating inclusion was measured in the fracture surface. In case of run out specimens, which did not fail after a fixed number of load cycles the stress intensity factor range cannot be calculated since the inclusion size is unknown. For that reason most run out specimens were fatigue tested again at a higher load amplitude. At higher loads the specimens fracture and the critical inclusion type and size can be observed in the fracture surface. With this information the stress intensity factor range, which led

* Corresponding author: schumacher@iwt-bremen.de 
previously to no fatigue failure, can be calculated. With this procedure the stress intensity factor ranges for fractured and run out specimens can be determined. However, the exact threshold stress intensity failure range $\Delta K_{\text {th }}$, which leads to a fatigue failure if this value is exceeded, cannot be measured directly at one single specimen. Generally, even specimens of the same batch have scattering mechanical properties so that a scattering threshold stress intensity failure range is expected as well. For a mathematical description of the scattering threshold value a two parametric Weibull distribution is used:

$$
P_{\mathrm{f}}=1-2^{-\left(\frac{\Delta K}{\Delta K_{\mathrm{th}}}\right)^{m}}
$$

With Equation 2 the fracture probability $P_{\mathrm{f}}$ of a specimen which is loaded with a stress intensity factor range $\Delta K$ can be calculated. The threshold stress intensity failure range $\Delta K_{\text {th }}$ describes the stress intensity factor range which leads to a fracture probability of $50 \%$ and the parameter $m$ is a measure for the scatter of the fatigue strength. Based on the experimental results the parameter $\Delta K_{\text {th }}$ and $m$ are determined by maximum likelihood estimation. In case of specimen failure a parameter set which maximizes the product of all fracture probabilities has to be determined. At the same time the product of survival probabilities $P_{\mathrm{s}}$ (Equation 3) of all run out specimens have to be maximized with the same parameter set.

$$
P_{\mathrm{S}}=1-P_{\mathrm{f}}
$$

Hence, the parameter combination of $\Delta K_{\text {th }}$ and $m$ which leads to maximum $L$ value is the best description of the experimental fatigue results:

$$
L\left(\Delta K_{\mathrm{th}}, m\right)=\prod_{\text {Fracture }} P_{\mathrm{f}} \cdot \prod_{\text {Run out }} P_{\mathrm{s}}
$$

According to Murakami and Yamashita [8] the threshold stress intensity failure range $\Delta K_{\text {th }}$ depends for an inclusion in the interior of the specimen on the Vickers hardness $H_{\mathrm{V}}$ of the steel and the inclusion size area $^{0.5}$ which causes the failure and can be calculated as follows:

$$
\Delta K_{\mathrm{th}}=0.00277 \cdot\left(H_{\mathrm{V}}+120\right) \cdot(\sqrt{\text { area }})^{1 / 3}
$$

In this empiric formula $\Delta K_{\text {th }}$ is expressed in terms of $\mathrm{MPa} \mathrm{m} \mathrm{m}^{0,5}$ and the area $^{0,5}$ in $\mu \mathrm{m}$. To take the effect of both the hardness and the inclusion size into account a similar approach was used in this investigation. As displayed in Equation 6 the parameters $C_{1}, C_{2}$ and $C_{3}$ have to be determined based on the experimental results.

$$
\Delta K_{\mathrm{th}}=C_{1} \cdot\left(H_{\mathrm{V}}+C_{2}\right) \cdot(\sqrt{\text { area }})^{C_{3}}
$$

Depending on the loading condition, mean stresses have to be considered at the fatigue strength evaluation. Multiaxial loading condition are possible or can occur in notches at uniaxial loading. Furthermore, multiaxial residual stresses caused by the specimen manufacturing or heat treatment exist in the highly stressed volume and have to be considered at the evaluation. Four different fatigue criteria were used in this investigation to take multiaxial stresses, mean stresses and residual stresses into account. The equivalent stress amplitude $\sigma_{\mathrm{ae}}$ has been calculated according to Bomas [9] (Equation 7), Crossland [10] (Equation 8), Dang Van [11] (Equation 9) and Sines [12] (Equation 10).

Bomas [9]: $\quad \sigma_{\mathrm{ae}}=2 \cdot\left(\tau_{\mathrm{a}, \max }+\alpha \cdot p_{\mathrm{m}}\right)$

Crossland [10]: $\quad \sigma_{\mathrm{ae}}=\frac{3}{\sqrt{2}+\alpha}\left(\tau_{\mathrm{a}, \mathrm{oct}}+\alpha \cdot p_{\max }\right)$

$\operatorname{Dang} \operatorname{Van}[11]: \quad \sigma_{\mathrm{ae}}=\frac{\left(\tau_{\mathrm{a}, \max }+\alpha \cdot p_{\max }\right)}{\frac{1}{2}+\frac{\alpha}{3}}$

Sines [12]: $\quad \sigma_{\mathrm{ae}}=\frac{3}{\sqrt{2}}\left(\tau_{\mathrm{a}, \mathrm{oct}}+\alpha \cdot p_{\mathrm{m}}\right)$

In the fatigue criteria of Bomas and Dang Van the maximum shear stress amplitude $\tau_{\mathrm{a}, \max }$ is required. Crossland and Sines are using the octahedral shear stress amplitude $\tau_{\mathrm{a}, \text { oct }}$ instead. Besides the stress amplitudes the hydrostatic stress state of the fatigue specimens are considered in all fatigue criteria. Bomas and Sines use the mean hydrostatic pressure $p_{\mathrm{m}}$. Crossland and Dang Van consider the maximum hydrostatic pressure $p_{\max }$. All fatigue criteria have in common that the parameter $\alpha$ has to be known in advance to calculate the equivalent stress amplitudes. This parameter depends on the material and is usually determined experimentally.

Schleicher et al. determined the parameter $\alpha$ for Dang Van's and Bomas' fatigue criterion in dependence of the Vickers hardness $H_{\mathrm{V}}$ of the steel [13]:

$$
\alpha=\frac{H_{\mathrm{V}}}{H_{\alpha}}
$$

In case of Dang Van's fatigue criterion the parameter $H_{\alpha}$ is $1263 \mathrm{HV}$, while in case of Bomas' fatigue criterion $H_{\alpha}$ is $1500 \mathrm{HV}$ [13]. In this approach the parameter $\alpha$ for the different fatigue criteria is correlated with the mean stress sensitivity $M$. Furthermore, a linear dependence of the mean stress sensitivity on the hardness is assumed, which can be described by the parameters $A$ and $B$ :

$$
M=A \cdot H_{\mathrm{V}}+B
$$

At a uniaxial loading condition, the mean stress sensitivity $M$ allows to calculate the fatigue limit $\sigma_{\mathrm{D}_{-}}\left(\sigma_{\mathrm{m}}\right)$ in dependence of the mean stress $\sigma_{\mathrm{m}}$ with knowledge of the mean stress free fatigue limit $\sigma_{\mathrm{W}}$. 


$$
\sigma_{\mathrm{D}}\left(\sigma_{\mathrm{m}}\right)=\sigma_{\mathrm{W}}-M \cdot \sigma_{\mathrm{m}}
$$

Under the assumption that this mean stress sensitivity approach works, this correlation can also be obtained at a uniaxial load case with the different fatigue criteria. For example at a uniaxial stress amplitude $\sigma_{\mathrm{a}}$ and a mean stress $\sigma_{\mathrm{m}}$ Bomas' fatigue criterion (Equation 7) results in the following expression:

$$
\sigma_{\mathrm{a}}=\sigma_{\mathrm{ae}}-\frac{2}{3} \cdot \alpha \cdot \sigma_{\mathrm{m}}
$$

Since the equivalent stress amplitude $\sigma_{\mathrm{ae}}$ corresponds to a mean stress free uniaxial loading condition it is comparable with the mean stress free fatigue limit $\sigma_{\mathrm{W}}$. In the event that Equation 13 and 14 are both valid, the following relationship between the mean stress sensitivity $M$ and the parameter $\alpha$ of Bomas' fatigue criterion exists:

Bomas: $\quad \alpha=\frac{3}{2} \cdot M=\frac{3}{2} \cdot A \cdot H_{\mathrm{V}}+\frac{3}{2} \cdot B$

By combining Equation 15 and 12 the parameter $\alpha$ can be expressed with the parameters $A$ and $B$ in dependence of the hardness of the steel specimens:

$$
\alpha=\frac{3}{2} \cdot A \cdot H_{\mathrm{V}}+\frac{3}{2} \cdot B
$$

The same approach was used to find a correlation between the mean stress sensitivity $M$ and the parameter $\alpha$ for the three other fatigue criteria used in this investigation. The correlation for Crossland's fatigue criterion is displayed in Equation 17, for the Dang Van's fatigue criterion in Equation 18 and for Sines' fatigue criterion in Equation 19.

Crossland: $\quad \alpha=\frac{\sqrt{2} \cdot A \cdot H_{\mathrm{V}}+\sqrt{2} \cdot B}{1-A \cdot H_{\mathrm{V}}-B}$

$$
\text { Dang Van: } \quad \alpha=\frac{\frac{3}{2} \cdot A \cdot H_{\mathrm{V}}+\frac{3}{2} \cdot B}{1-A \cdot H_{\mathrm{V}}-B}
$$

Sines:

$$
\alpha=\sqrt{2} \cdot A \cdot H_{\mathrm{V}}+\sqrt{2} \cdot B
$$

\section{Results and discussion}

As described before, the parameters $C_{1}, C_{2} C_{3}, A, B$ and $m$ are required to describe the fatigue behaviour in case of an inclusion failure. The fatigue test results of case hardened, quenched and tempered as well as bearing steels in different heat treatment conditions, which have been investigated in several research projects over the last two decades, are used as database. Only fatigue specimens which failed at an inclusion were suitable for this investigation. A total of 603 specimens were taken into account in the parameter determination. Oxides, sulphides, carbides or nitrides were the failure causing inclusions. The local stress amplitudes and mean stresses at the position of the fracture causing inclusion have to be calculated. In case of notched specimens or complex loading conditions the entire multiaxial stress state has been regarded. Furthermore, the residual stresses and the hardness at the crack initiation site have been determined. The entire database is displayed exemplarily in Figure 1.

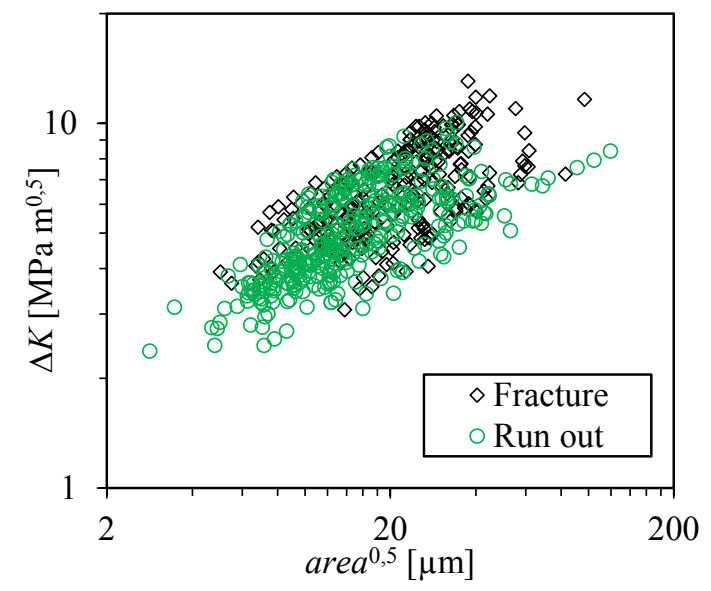

Fig. 1. Specimen used as database for the adaption of the parameters. Dang Van's fatigue criterion was used for the calculation of $\Delta K$.

The diagram shows the stress intensity factor range $\Delta K$ according to Murakami (Equation 1) of the fractured and run out specimens in dependence of the size area $a^{0,5}$ of the failure causing inclusion. For the calculation of the equivalent stress amplitude $\sigma_{\mathrm{ae}}$ the fatigue criterion of Dang Van (Equation 9) was used and the parameter $\alpha$ was calculated according to Schleicher et al. (Equation 11) with a $H_{\alpha}$ of $1263 \mathrm{HV}$ [13]. The inclusion size ranges between 2.8 and $119 \mu \mathrm{m}$. Since the hardness of the investigated specimens ranged between 357 and 776 $\mathrm{HV}$ the stress intensity factor range $\Delta K$, which leads to a fracture or a run out specimen, is scattering as well. Nevertheless, an increase of the stress intensity factor range $\Delta K$ with increasing inclusion size is visible. However, it must be considered, that the stress intensity factor range $\Delta K$ is influenced by the chosen fatigue criterion.

\subsection{Influence of the inclusion type on the fatigue strength}

For a detailed investigation of the influence of the inclusion type on the fatigue strength, steel batches with a constant hardness and different crack initiating inclusions have been analysed. In that case the influence of the hardness on the fatigue strength did not superimpose the result. Exemplarily, Figure 2 shows the fatigue test results of two bearing steel batches. Figure 2 a shows batch A with a hardness of $765 \mathrm{HV}$ and Figure 2 b shows batch B with a hardness of $756 \mathrm{HV}$. Both batches have been tested at push-pull-loading at the stress ratio $R=-1$. Additionally, batch $\mathrm{A}$ was tested at alternating tensile loading at the stress ratio of $R=0.1$ 
and batch $\mathrm{B}$ was tested at alternating tensile loading at the stress ratio $R=0$.

In batch $\mathrm{A}$ oxides and carbides are the dominant failure types. One failure at a sulphide and some fractures at nitrides occur as well. However, the number of crack initiations at nitrides is significantly higher at $R$ $=0.1$ than at $R=-1$. On average, the oxide inclusion are larger than the nitrides, carbides or the sulphides. For the same inclusion size the stress intensity factor range $\Delta K$ is higher at a stress ratio of $R=-1$ compared to $R=0.1$. This gives an indication that the the different loading conditions are described inaccurately by the fatigue criterion. At both stress ratios the stress intensity factor range increases with increasing inclusion size. However, an influence of the inclusion type on the fatigue strength is not visible.
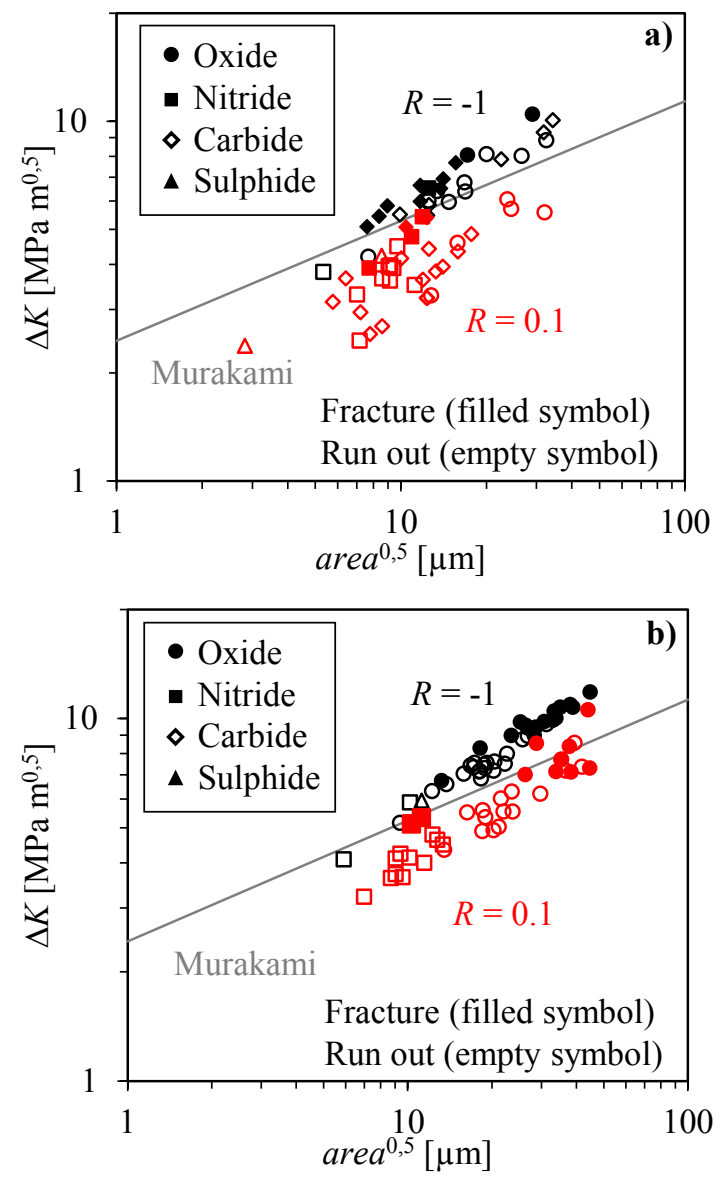

Fig. 2. Stress intensity factor range at different inclusion types in fractured and run out specimens of a) batch A and b) batch B.

At batch $\mathrm{B}$ oxides are the dominant failure type. Nitrides occur as failure causes as well, especially at $R=0$. The average size of the oxides is larger than that of the nitrides. At the same inclusion size the stress intensity factor range $\Delta K$ is higher at $R=1$ compared to $R=0$. This gives again an indication that the fatigue criterion describes the different loading conditions inaccurately. At both stress ratios the stress intensity factor range increases with increasing inclusion size. Again, an influence of the inclusion type on the fatigue strength is not visible.
The prediction of the threshold stress intensity factor range $\Delta K_{\text {th }}$ according to Murakami and Yamashita [8] (Equation 5) is displayed for both steel batches in Figure $2 \mathrm{a}$ and $\mathrm{b}$ as well. For both batches the prediction separates the values at push pull and alternating tensile loading. Since the predicted threshold stress intensity factor range $\Delta K_{\text {th }}$ describes the $50 \%$ fracture probability of the fatigue specimens the line should be between the fractured and run out specimens. Both the chosen fatigue criterion as well as the predicted threshold stress intensity failure range can be responsible for this deviation. Furthermore, the experimental stress intensity factor range values increase more strongly with increasing inclusion size than the predicted threshold stress intensity factor range.

In summary different failure types lead to fatigue failure of the investigated specimens. Depending on the inclusion type different average inclusion sizes are observed. Taking into account the influence of the inclusion size on the fatigue strength, no difference between the inclusion types is recognizable. For the calculation of the fatigue strength follows, that no distinction has to be made between the inclusion types.

\subsection{Influence of the inclusion size, hardness and loading condition on the fatigue strength}

The entire database displayed in Figure 1 is used to determine the most likely parameters $C_{1}, C_{2} C_{3}, A, B$. The parameter $m$ depends on the individual scatter of each batch and cannot be used for a prediction of the scatter of other batches. For that reason the parameter $m$ was set to 20 for the fitting procedure. The parameter sets for the four fatigue criteria which lead to the maximum likelihood values according Equation 4 are displayed in Table 1.

Table 1. Parameter $C_{1}, C_{2}, C_{3}, A$, and $B$ determined at the entire database for the four investigated fatigue criteria.

\begin{tabular}{|c|c|c|c|c|c|}
\hline $\begin{array}{c}\text { Fatigue } \\
\text { criterion }\end{array}$ & $C_{1}$ & $C_{2}$ & $C_{3}$ & $A$ & $B$ \\
\hline Bomas & 0.00290 & 95 & 0.384 & 0.00128 & -0.383 \\
\hline Crossland & 0.00287 & 100 & 0.382 & 0.00120 & -0.343 \\
\hline Dang Van & 0.00285 & 104 & 0.383 & 0.00122 & -0.359 \\
\hline Sines & 0.00291 & 94 & 0.382 & 0.00125 & -0.358 \\
\hline
\end{tabular}

There are no major differences between the parameter sets for the different fatigue criteria. The resulting mean stress sensitivity $M$ and the weighting factor $\alpha$, which are both calculated based on the parameters $A$ and $B$, are displayed as a function of the hardness in Figure 3 and Figure 4, respectively.

For all fatigue criteria, an almost identical trend of the mean stress sensitivity depending on the hardness can be observed. The curves show an increase of the mean stress sensitivity with increasing hardness. The 
weighting factor $\alpha$ shows, depending on the chosen fatigue criterion, a different curve shape. For Bomas' and Sines' fatigue criterion the curves increase linear with increasing hardness. For both fatigue criteria the weighting factor is nearly identical. For Crossland's and Dang Van's criterion a progressive increase of the weighting factor $\alpha$ with increasing hardness was determined. For these two fatigue criteria the weighting factors are nearly identical as well.

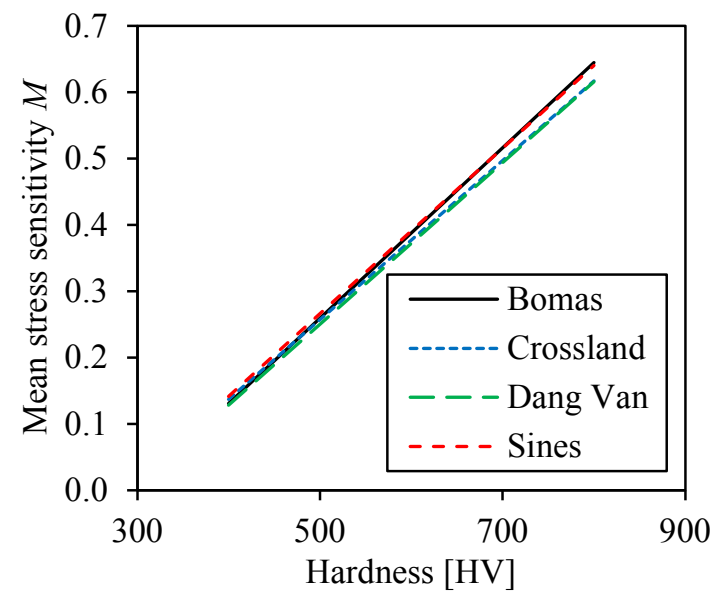

Fig. 3. Mean stress sensitivity $M$ determined with different fatigue criteria as a function of the specimen hardness.

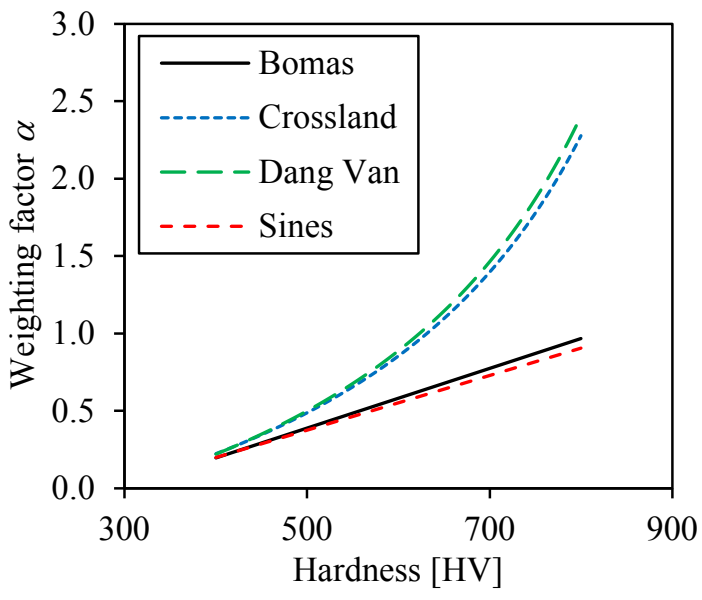

Fig. 4. Weighting factor $\alpha$ determined for the different fatigue criteria as a function of the specimen hardness.

Figure 5 shows the influence of the hardness on the threshold stress intensity factor range $\Delta K_{\text {th }}$ exemplarily for an inclusion size of $50 \mu \mathrm{m}$. All investigated fatigue criteria lead to nearly identical threshold values. Compared to the threshold value according to Murakami (Equation 5) the calculated threshold values are located above the Murakami curve and the increase with hardness is somewhat steeper.

Both resulting parameters, the mean stress sensitivity $M$ and the threshold stress intensity factor range $\Delta K_{\text {th }}$ are nearly identical for the different fatigue criteria investigated. However, it should be noted that a large number of the specimens examined were subjected to uniaxial tensile compression or alternating tensile loads.
In combination with the chosen calculation approach for weighting parameter $\alpha$, the four investigated fatigue criteria lead to the same equivalent stress amplitude in case of uniaxial tensile compression or alternating tensile loads. Due to the small number of multi-axially loaded samples, the determined parameters for the different fatigue criteria are close to each other.

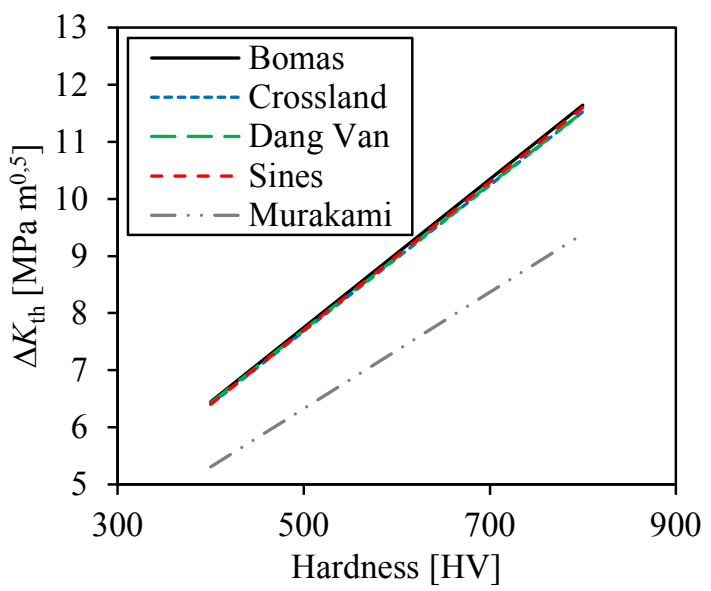

Fig. 5. Threshold stress intensity factor range determined for different fatigue criteria and threshold stress intensity factor range according to Murakami as a function of hardness displayed for an inclusion size of $50 \mu \mathrm{m}$.

To show the influence of the new parameters the data of the batches A and B, which are displayed in Figure 2, are used. As an example, the parameters of Bomas' fatigue criterion are used and displayed in Figure 6. 

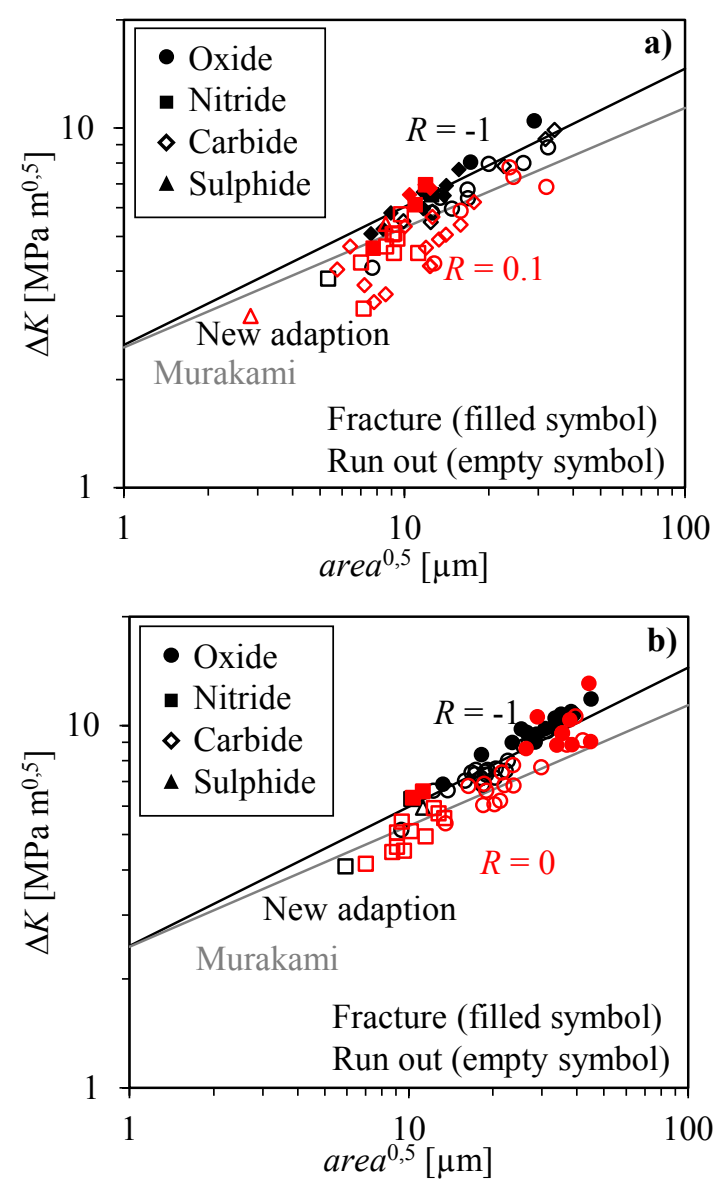

Fig. 6. Stress intensity factor range calculated with Bomas' fatigue criterion with the parameters, $A$ and $B$ of Table 1 in fractured and run out specimens of a) batch A and b) batch B. The threshold stress intensity range values have been calculated according to Murakami and the new adaption for Bomas' fatigue criterion.

Compared with the results displayed in Figure 2 the gap between the specimens tested with and without mean stress disappears with the new calculation approach.

The determined threshold stress intensity factor range and the threshold value according to Murakami are shown in Figure 6 as well. In case of small inclusion sizes, both approaches supply almost identical values for the threshold stress intensity factor range. In the new approach, the threshold stress intensity factor range increases more strongly with increasing inclusion size, so that a higher threshold stress intensity factor range is calculated with this approach for large inclusions. Since both threshold stress intensity factor range calculation approaches only depend on the size of the inclusion and the hardness of the specimen not all fatigue strength determining factors are considered. Therefore, the threshold stress intensity factor range of different steel batches is described with varying degrees of accuracy. For example, the threshold value according to Murakami separates the fractured and run out specimens of batch A slightly more accurate than the threshold value calculated in this investigation, as can be seen in Figure 6 a. In comparison, the newly calculated threshold value describes the data of batch $\mathrm{B}$ much more precisely than the threshold value of Murakami, as can be seen in Figure $6 \mathrm{~b}$.

\section{Summary}

An empiric fracture mechanical approach is presented describing the fatigue strength of high strength steel specimens. This calculation method combines the approach of Murakami and different fatigue criteria assuming that inclusions are responsible for the fatigue failure. The hardness of the specimens and the crack initiating inclusion sizes are the fatigue strength determining material parameters. The required model parameter have been determined at 603 fatigue specimens which have been tested in several research projects over the last two decades and failed at inclusions.

Different inclusion types like oxides, carbides, nitrides or sulphides showed the same effect on the fatigue strength. Hence the calculation approach does not distinguish between different inclusion types.

Based on the experimental data the threshold stress intensity factor range for a fracture probability of $50 \%$ was determined. Compared with the threshold value according to Murakami the new approach results in a slightly higher threshold value and a steeper increase of the threshold stress intensity factor range with increasing inclusion size.

Additionally, a calculation approach for the material depending weighting factor $\alpha$ of the different fatigue criteria is described. Individually, for each fatigue criterion the weighting factor can be determined on the basis of the mean stress sensitivity.

In summary, with the knowledge of the hardness and the expected failure-critical inclusion size, the introduced calculation approach enables the prediction of the fatigue strength for arbitrary steel batches and loading conditions.

The IGF Project $(18973 \mathrm{~N})$ of the Arbeitsgemeinschaft Wärmebehandlung und Werkstofftechnik e. V. (AWT) was funded by the Arbeitsgemeinschaft industrieller Forschungsvereinigungen „Otto von Guericke“ e. V. (AiF) through the IGF program of the Bundesministerium für Wirtschaft und Energie (BMWi) due to a resolution of the Deutscher Bundestag. The authors are grateful for the financial support. We further wish to thank the Expert Committee 21 "Microstructure and mechanical properties of heat treated materials" of AWT for scientific support and supervision of the project. Our special thank is given to the members of the Board of Experts for their collaboration and interest in the project: Deutsche Edelstahlwerke GmbH, Georgsmarienhütte $\mathrm{GmbH}$, Robert Bosch GmbH, SchaefflerTechnologies AG \& Co.KG, Steinhoff $\mathrm{GmbH} \& \mathrm{Cie}$. OHG, Thermo Fisher Scientific $\mathrm{GmbH}$ and ZF-Friedrichshafen AG.

\section{References}

1. J. Rösler, H. Harders, M. Bäker, Mechanisches Verhalten der Werkstoffe (Vieweg + Teubner Verlag, Wiesbaden, 2008) 
2. A. Melander, M. Rolfsson, A. Nordgren, B. Jansson, H. Hedberg, T. Lund, Scand. J. Metall. 20 (1991)

3. H. Bomas, M. Schleicher, Fatigue Fract. Eng. Mater. Struct. 28, 11 (2005)

4. H. Bomas, M. Bacher-Hoechst, R. Kienzler, S. Kunow, G. Loewisch, F. Muehleder, R. Schroeder,. Fatigue Fract. Eng. Mater. Struct. 33, 2 (2010)

5. J. Ma, B. Zhang, D. Xu, E.-H. Han, W. Ke, Int. J. Fatigue 32 (2010)

6. Y. Murakami, Metal Fatigue: Effects of Small Defects and Nonmetallic Inclusions (Elsevier, Oxford 2002)

7. J. Monnot, R. Heritier, J. Y. Cogne, Effect of Steel Manufacturing Processes on the Quality of Bearing Steels (ASTM STP 987, 1988)

8. Y. Murakami, Y. Yamashita, Procedia Eng. 74 (2014)

9. H. Bomas, T. Linkewitz, P. Mayr, Fatigue Fract. Eng. Mater. Struct. 22 (1999)

10. B. Crossland, Proceedings of the International Conference on the Fatigue of Metals (Institute of Mechanical Engineers, London, 1956)

11. K. Dang Van, B. Griveau, O. Message, Biaxial and Multiaxial Fatigue (EGF 3) (Mechanical Engineering Publications, London, 1989)

12. G. Sines, Metal Fatigue (McGraw Hill, New York, 1959)

13. M. Schleicher, H. Bomas, P. Mayr, HTM HaertereiTechn. Mitt., 56 (2001) 